\title{
Primary familial brain calcification in a patient with a novel compound heterozygous mutation in MYORG presenting with an acute ischemic stroke: a case report
}

\author{
Qijie Yang ${ }^{1 \#}$, Jian $^{\mathrm{Li}^{2 \#}}$, Bin Jiao ${ }^{1,3}$, Ling Weng ${ }^{1,3}$ \\ ${ }^{1}$ Department of Neurology, Xiangya Hospital, Central South University, Changsha, China; ${ }^{2}$ Department of Neurosurgery, Xiangya Hospital, Central \\ South University, Changsha, China; ${ }^{3}$ National Clinical Research Center for Geriatric Disorders, Central South University, Changsha, China \\ \#These authors contributed equally to this work. \\ Correspondence to: Ling Weng. Department of Neurology, Xiangya Hospital, Central South University, 87 Xiangya Road, Changsha, China. \\ Email: lingweng@csu.edu.cn.
}

\begin{abstract}
Primary familial brain calcification (PFBC) is known as Fahr's disease (FD) or familial idiopathic basal ganglia calcification (FIBGC). PFBC is a genetically heterogeneous disease characterized by extensive intracranial calcium deposition. Currently, pathogenic variants in six genes (SLC20A2, PDGFB, PDGFRB, $X P R 1, M Y O R G$ and $7 A M 2$ ) have been associated with PFBC. MYORG was the first autosomal-recessive causal gene discovered in PFBC patients. $\mathrm{PFBC}$ is also a clinically heterogeneous disorder. Patients mostly present with movement disorders, cognitive impairment and psychiatric symptoms, and acute cerebrovascular attacks are rare. Here, we report the case of a PFBC patient with a novel compound heterozygous mutation in MYORG presenting with an acute ischemic stroke. A 52-year-old man had recurrent and progressively exacerbated transient-ischemic-attack-like episodes and finally had an acute ischemic stroke. Brain computed tomography (CT) showed extensive and symmetric calcifications. Brain magnetic resonance imaging (MRI) revealed an acute ischemic infarction. A novel compound heterozygous mutation in MYORG (p.R116_ S117insLAFR and p.Q445*) was found in this patient by whole-exome sequencing (WES). Therefore, this patient was diagnosed with PFBC-MYORG and an acute ischemic stroke. He was treated with antiplatelet drugs (aspirin and clopidogrel) and received rehabilitation training. There was no physical disability at discharge. More efforts should be made to explore the association between acute ischemic strokes and PFBC.
\end{abstract}

Keywords: MYORG; primary familial brain calcification (PFBC); acute ischemic stroke; Fahr's disease (FD); case report

Submitted Sep 14, 2021. Accepted for publication Dec 14, 2021.

doi: 10.21037/atm-21-4883

View this article at: https://dx.doi.org/10.21037/atm-21-4883

\section{Introduction}

Primary familial brain calcification (PFBC) is a rare neurodegenerative disorder also known as Fahr's disease (FD) or familial idiopathic basal ganglia calcification (FIBGC). PFBC is characterized by extensive calcium deposition, including in the basal ganglia, cerebellum, thalamus, brainstem, and subcortical white matter (1). $\mathrm{PFBC}$ is a genetically heterogeneous disorder. To date, pathogenic variants in four genes (SLC20A2, PDGFB, $P D G F R B$ and XPR1) have been associated with autosomal- dominant PFBC (AD-PFBC). MYORG and $\mathcal{F} A M 2$ were identified as the causative genes of PFBC in 2018 and 2020, respectively. Pathogenic variants in $M Y O R G$ and FAM2 have been associated with autosomal-recessive PFBC (AR-PFBC) $(2,3)$. PFBC is also a clinically heterogeneous disorder. A systematic review found that one-third of PFBC patients were clinically unaffected, and the combination of motor and nonmotor presentations was most common in clinically affected patients (4). Typical symptoms of PFBC occur at the age of 30 to 60 years (1). Symptoms are 


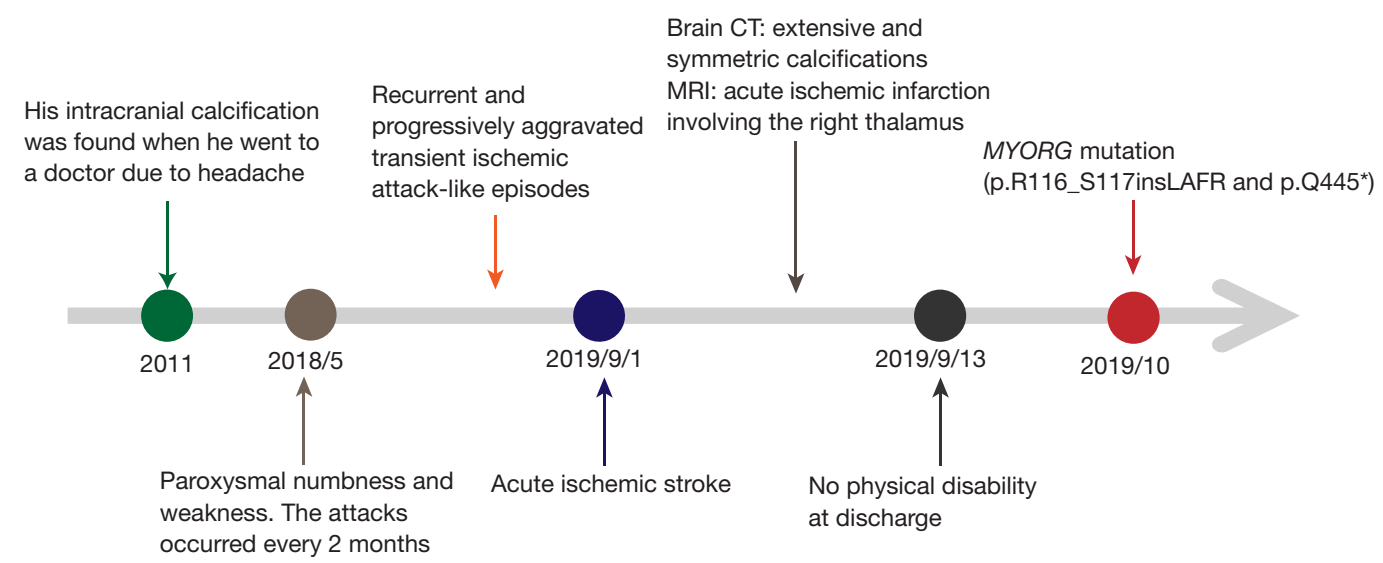

Figure 1 Timeline of major clinical events in the patient. CT, computed tomography; MRI, magnetic resonance imaging.

generally limited to the nervous system, including basal ganglia movement disorder, pyramidal signs, cognitive impairment, gait disorder, cerebellar abnormalities, speech dysfunction, psychiatric presentations, sensory changes and others (5). However, acute cerebrovascular attacks in PFBC patients are rare (6-14). Here, we report the first case of PFBC in which the patient had a novel compound heterozygous mutation in $M Y O R G$ and presented with an acute ischemic stroke. We present the following article in accordance with the CARE reporting checklist (available at https://atm.amegroups.com/article/view/10.21037/atm-21$4883 / \mathrm{rc})$.

\section{Case presentation}

A 52-year-old man was admitted to the hospital complaining of recurrent left limb weakness for more than 1 year and exacerbation for 2 days. No family history of genetic diseases or stroke was identified.

At first, he complained of left upper limb weakness and numbness, which lasted 1 to 2 hours and then spontaneously recovered. The attacks occurred every 2 months. Gradually, the regions of recurrent weakness expanded, and his left upper and lower limbs were both involved in the attacks. The episodes occurred more frequently, once every 8 to 10 days. The last attack was long in duration, and the symptoms improved slowly even with medical treatment. Two days later, another attack occurred. The weakness in the left limb was worse than before and gradually exacerbated (Figure 1). Intracranial calcifications were found in a computed tomography (CT) scan 8 years ago when he went to a doctor due to a headache. There were no other neurological symptoms except for stroke and headache.

Physical examination revealed decreased muscle power (grade 4), hypotonia, decreased reflexes, and superficial sensory disturbances in the left upper and lower limbs. Pathological reflexes and cerebellar ataxia were absent. His National Institutes of Health Stroke Scale (NIHSS) score was 3 . He reported neither smoking nor drinking.

At hospitalization, we investigated the possible causes of cerebral infarction and abnormal intracranial calcifications. The levels of serum calcium $(2.18 \mathrm{mmol} / \mathrm{L})$, phosphorus $(0.91 \mathrm{mmol} / \mathrm{L})$, thyroid hormone and human immunodeficiency virus (HIV) were within the normal range. 25 -Hydroxyvitamin $\mathrm{D}(25.27 \mathrm{pg} / \mathrm{mL})$ was slightly reduced, and parathyroid hormone $(118.9 \mathrm{pg} / \mathrm{mL})$ was slightly increased. Without special treatment, we performed 24-hour urine electrolyte analysis, and urinary calcium $(2.4 \mathrm{mmol} / 24 \mathrm{~h})$ and potassium $(7.86 \mathrm{mmol} / 24 \mathrm{~h})$ were both decreased. Cranial CT showed extensive and symmetric calcifications involving the bilateral centrum semiovale, corona radiata, paraventricular, basal ganglia, thalami, vermis cerebelli, cerebellar hemispheres and midbrain (Figure 2). Brain magnetic resonance imaging (MRI) revealed acute ischemic infarction involving the right thalamus (Figure 2F). Intracranial magnetic resonance angiography (MRA) excluded intracranial large-vessel stenosis, aneurysm and vascular malformation. Contrastenhanced MRI excluded abnormal parenchymal or leptomeningeal enhancement. Carotid artery computed tomography angiography (CTA) excluded aortic arch plaques. The stroke surveys, including low-density lipoprotein, homocysteine, coagulation function tests; a carotid artery duplex scan; a transcranial Doppler 

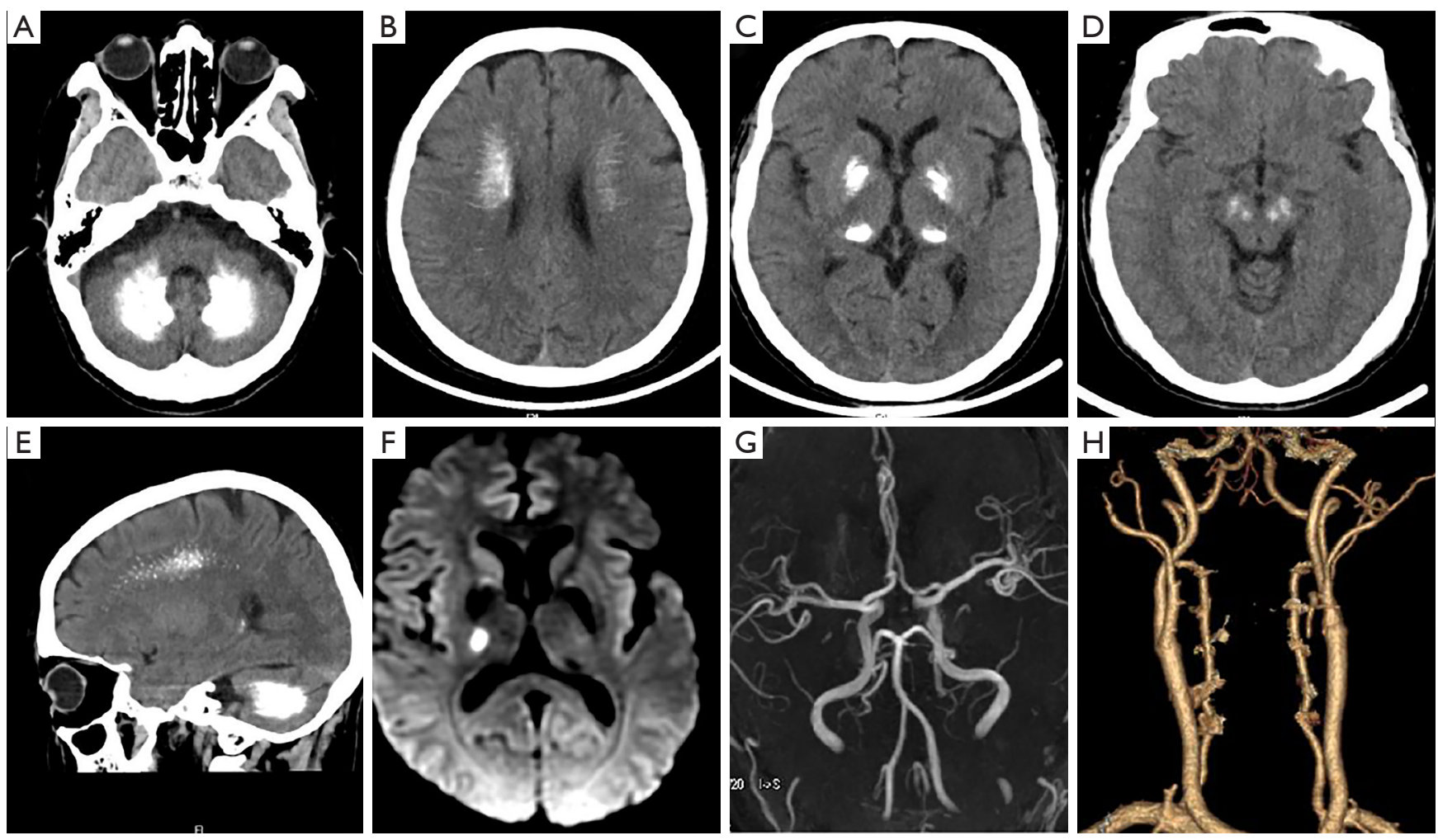

Figure 2 Imaging examination. (A-D) CT scans show calcifications in the cerebellum, periventricular white matter, basal ganglia, thalamus and brainstem; (E) CT scans show the cerebellum with subtle antero-superior hemispheric lobe atrophy; (F) MRI DWI shows acute ischemic infarction of the right thalamus; $(\mathrm{G}, \mathrm{H})$ MRA and CTA exclude intracranial and extracranial vessel stenosis, occlusion, aneurysm and vascular malformation. CT, computed tomography; MRI, magnetic resonance imaging; DWI, diffusion weighted image; MRA, magnetic resonance angiography; CTA, computed tomography angiography.

(TCD) bubble study; a heart Doppler ultrasound; and electrocardiography, showed no abnormalities. We found a novel compound heterozygous mutation in MYORG in this patient by whole-exome sequencing (WES). One mutation was a heterozygous nonsense mutation (rs1563981743, c.1333C>T; p.Q445*), and the other mutation was a nonframeshift indel (rs775762093, c.348_349insCTGGCCTTCCGC; p.R116_S117insLAFR) (Figure 3).

Therefore, the patient was diagnosed with PFBC and an acute ischemic stroke. He was treated with antiplatelet drugs (aspirin and clopidogrel) and received rehabilitation training. He had no physical disability at discharge.

All procedures performed in this study were in accordance with the ethical standards of the institutional and/or national research committee(s) and with the Helsinki Declaration (as revised in 2013). Written informed consent was obtained from the patient for the publication of this case report and accompanying images. A copy of the written consent is available for review by the editorial office of this journal.

\section{Discussion}

The MYORG gene is the first PFBC-causative gene with autosomal-recessive inheritance. Progressive movement disorders, such as speech disturbance, bradykinesia and ataxia, from middle age onward are the most common symptoms in PFBC-MYORG (4). The relatively characteristic imaging features of PFBC-MYORG are pontine calcifications and cerebellar atrophy (vermis or antero-superior lobe) (15).

The patient had extensive and symmetric intracranial calcifications involving the brainstem. There was no obvious secondary cause, such as intracranial infection, immunodeficiency, intrauterine or perinatal infection, abnormal growth and development, toxic exposure, 


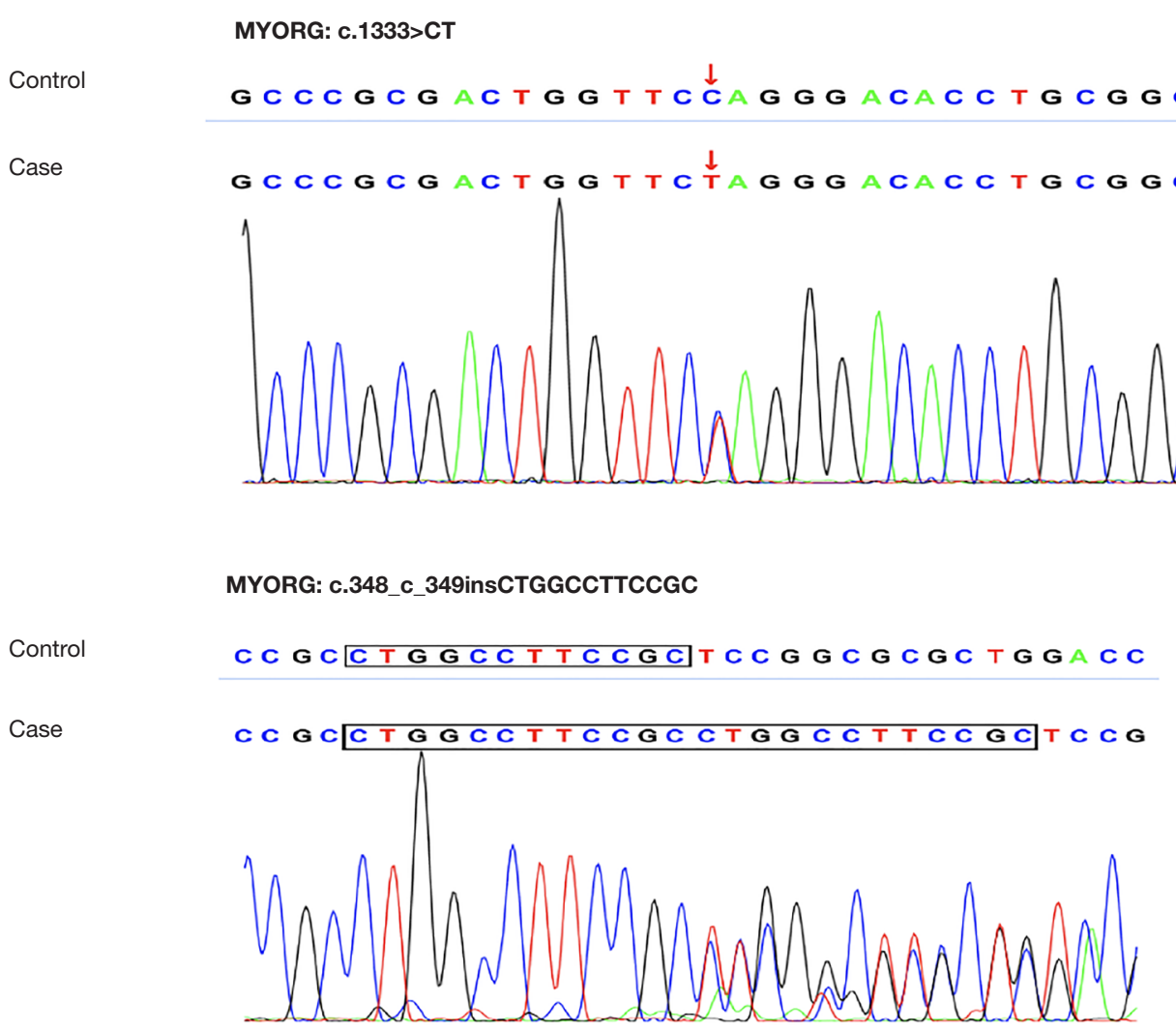

Figure 3 Sequence analysis indicates a nonsense mutation (c.1333C>T) and nonframeshift indel variant (c.348_349insCTGGCCTTCCGC). Control, reference sequence; case, sequence of the patient.

hypoparathyroidism or hyperparathyroidism. The slight increase in parathyroid hormone was due to insufficient 25-hydroxyvitamin D rather than hyperparathyroidism because phosphate and calcium levels were in the normal range. By the recommendation of the American College of Medical Genetics (ACMG), c.1333C>T was categorized as a "pathogenic variant" (because of its classification as PVS1 + PM2 + PP3 + PP5), and the c.348_349insCTGGCCTTCCGC variant was categorized as a "likely pathogenic variant" (because of its classification as $\mathrm{PM} 2+\mathrm{PM} 4+\mathrm{PP} 3+\mathrm{PP} 5)$. Both the homozygous c.348_349insCTGGCCTTCCGC variant (p.R116_ S117insLAFR) and the homozygous c.1333C $>\mathrm{T}$ variant (p. Q445*) have been reported in PFBC patients $(16,17)$. Thus, this patient had PFBC.

This patient had transient-ischemic-attack-like episodes that increased in frequency and duration and finally had an acute ischemic stroke. There were no risk factors for stroke, such as smoking, high blood pressure, coagulopathy, intracranial and extracranial atherosclerotic stenosis or occlusion, an abnormal cardiac structure or rhythm or microemboli. According to the Trial of Org 10172 in Acute Stroke Treatment (TOAST) classification system, we classified the stroke as being from an undetermined cause. Therefore, this patient was diagnosed with PFBC and an acute ischemic stroke.

We report the first case of PFBC in which the patient had a novel compound heterozygous mutation in MYORG and presented with an acute ischemic stroke. The neurovascular unit (NVU) plays an important role in PFBC and is composed of astrocytes, endothelial cells, pericytes, neurons, etc. (18). The six pathogenic genes have a diverse relationship with the NVU. PDGFB and PDGFRB are necessary for angiogenesis and proper formation of the blood-brain barrier (19). MYORG in mice is specifically expressed in astrocytes (2). Future studies are needed to investigate the relationship of the NVU with PFBC.

Moreover, acute ischemic strokes have been reported in PFBC patients $(6,11,14)$. Interestingly, a patient $(990-$ 002) with a biallelic MYORG variant (p.Gly64Glu) had 
multiple ischemic strokes (right cerebellar territory and left middle cerebral artery territory) (14). Clinically, bilateral anteroposterior circulation cerebral infarction is most common in cardiogenic embolism and hypoperfusion. Grangeon et al. classified the stroke as a cardiogenic embolism (14). In our opinion, we cannot rule out the possibility of hypoperfusion. Some evidence shows that intracranial vascular calcifications are significant risk factors for acute ischemic strokes (20). One possible explanation is that intracranial calcifications led to small-vessel calcifications. Calcium can be deposited in small blood vessels, making the walls of the blood vessels thicker and less elastic. First, a thickened blood vessel wall may hinder substance exchange between the bloodstream and neurons, resulting in a relatively low perfusion state of local neurons. Moreover, a loss of vascular wall elasticity may cause hemodynamic changes, such as reduced blood velocity, blood flow stasis and thrombosis.

We report the case of a PFBC patient with a novel compound heterozygous mutation in MYORG presenting with TIA-like episodes that increased in frequency and duration and an acute ischemic stroke. More efforts should be made to explore the association between acute ischemic strokes and PFBC.

\section{Acknowledgments}

Funding: This research was supported by the National Natural Science Foundation of China (No. 81902551).

\section{Footnote}

Reporting Checklist: The authors have completed the CARE reporting checklist. Available at https://atm.amegroups. com/article/view/10.21037/atm-21-4883/rc

Conflicts of Interest: All authors have completed the ICMJE uniform disclosure form (available at https://atm. amegroups.com/article/view/10.21037/atm-21-4883/coif). The authors have no conflicts of interest to declare.

Ethical Statement: The authors are accountable for all aspects of the work in ensuring that questions related to the accuracy or integrity of any part of the work are appropriately investigated and resolved. All procedures performed in this study were in accordance with the ethical standards of the institutional and/or national research committee(s) and with the Helsinki Declaration (as revised in 2013). Written informed consent was obtained from the patient for the publication of this case report and accompanying images. A copy of the written consent is available for review by the editorial office of this journal.

Open Access Statement: This is an Open Access article distributed in accordance with the Creative Commons Attribution-NonCommercial-NoDerivs 4.0 International License (CC BY-NC-ND 4.0), which permits the noncommercial replication and distribution of the article with the strict proviso that no changes or edits are made and the original work is properly cited (including links to both the formal publication through the relevant DOI and the license). See: https://creativecommons.org/licenses/by-nc-nd/4.0/.

\section{References}

1. Ramos EM, Oliveira J, Sobrido MJ, et al. Primary Familial Brain Calcification. In: Adam MP, Ardinger HH, Pagon RA, et al., editors. GeneReviews ${ }^{\circledR}$. Seattle (WA): University of Washington, Seattle, 2004.

2. Yao XP, Cheng X, Wang C, et al. Biallelic Mutations in MYORG Cause Autosomal Recessive Primary Familial Brain Calcification. Neuron 2018;98:1116-1123.e5.

3. Schottlaender LV, Abeti R, Jaunmuktane Z, et al. Biallelic JAM2 Variants Lead to Early-Onset Recessive Primary Familial Brain Calcification. Am J Hum Genet 2020;106:412-21.

4. Balck A, Schaake S, Kuhnke NS, et al. GenotypePhenotype Relations in Primary Familial Brain Calcification: Systematic MDSGene Review. Mov Disord 2021;36:2468-80.

5. Perugula ML, Lippmann S. Fahr's Disease or Fahr's Syndrome? Innov Clin Neurosci 2016;13:45-6.

6. Zhang X, Ma G, Zhao Z, et al. SCL20A2 mutation presenting with acute ischemic stroke: a case report. BMC Neurol 2018;18:11.

7. Sarigul B, Seker S, Celik SE. Anterior communicating artery aneurysm in a young patient with Fahr's disease: case presentation. Br J Neurosurg 2019. [Epub ahead of print]. doi: 10.1080/02688697.2019.1690128.

8. Al-Jehani H, Ajlan A, Sinclair D. Fahr's Disease Presenting with Aneurysmal Subarachnoid Hemorrhage. J Clin Imaging Sci 2012;2:27.

9. Sgulò FG, di Nuzzo G, de Notaris M, et al. Cerebrovascular disorders and Fahr's disease: Report of two cases and literature review. J Clin Neurosci 2018;50:163-4. 
10. Eroglu U, Kahilogullari G, Demirel A, et al. Fahr's Syndrome Associated with Multiple Intracranial Aneurysms: A Case Report. Turk Neurosurg 2016;26:643-5.

11. Yang CS, Lo CP, Wu MC. Ischemic stroke in a young patient with Fahr's disease: a case report. BMC Neurol 2016;16:33.

12. Bartecki BF, Kamienowski J. Transient focal ischemia in Fahr's disease. Neurol Neurochir Pol 1979;13:443-7.

13. Asensio Moreno C, Arias Jiménez JL, Aramburu Bodas O, et al. Transient ischemic attack associated with a calcinosis cerebri syndrome. An Med Interna 2008;25:33-5.

14. Grangeon L, Wallon D, Charbonnier C, et al. Biallelic MYORG mutation carriers exhibit primary brain calcification with a distinct phenotype. Brain 2019;142:1573-86.

15. Nicolas G, Grangeon L, Wallon D. Reply: New homozygous indel in MYORG linked to brain calcification, thyroidopathy and neuropathy. Brain 2019;142:e52.

16. Chen Y, Fu F, Chen S, et al. Evaluation of MYORG mutations as a novel cause of primary familial brain calcification. Mov Disord 2019;34:291-7.

17. Peng $Y$, Wang $P$, Chen Z, et al. A novel mutation in MYORG causes primary familial brain calcification with central neuropathic pain. Clin Genet 2019;95:433-5.

18. Alvarez-Fischer D, Westenberger A. Biallelic MYORG mutations: Primary familial brain calcification goes recessive. Mov Disord 2019;34:322.

19. Betsholtz C, Keller A. PDGF, pericytes and the pathogenesis of idiopathic basal ganglia calcification (IBGC). Brain Pathol 2014;24:387-95.

20. Quiney B, Ying SM, Hippe DS, et al. The Association of Intracranial Vascular Calcification and Stenosis With Acute Ischemic Cerebrovascular Events. J Comput Assist Tomogr 2017;41:849-53.
Cite this article as: Yang Q, Li J, Jiao B, Weng L. Primary familial brain calcification in a patient with a novel compound heterozygous mutation in MYORG presenting with an acute ischemic stroke: a case report. Ann Transl Med 2022;10(7):423. doi: $10.21037 /$ atm-21-4883 